\title{
Bedeutung der Kompatibilität für eine sichere und belastungsfreie Arbeitsgestaltung - Aktuelle Relevanz des Konstruktes am Beispiel moderner Arbeitsgestaltung
}

\author{
Oliver Sträter ${ }^{1}$ \\ Angenommen: 1. September 2021 / Online publiziert: 24. Januar 2022 \\ (c) Der/die Autor(en) 2022
}

\section{Zusammenfassung}

Kompatibilität ist eines der zentralen Konzepte der Arbeitsgestaltung seit den frühen Anfängen arbeitswissenschaftlicher Forschung. Auch wenn, oder gerade weil, das Konstrukt der Kompatibilität etabliert ist, tritt die Bedeutung der Kompatibilität für aktuelle Fragestellungen der Arbeitsgestaltung jedoch oft in den Hintergrund, weil es ein altes und damit vermeintlich antiquiertes Konstrukt ist. Gerade der Umstand, dass Kompatibilität ganz wesentlich physiologisch, also durch unsere Hirnanatomie bedingt ist, zeigt jedoch, dass genau das Gegenteil der Fall ist. Die Bedeutung der Kompatibilität für aktuelle Fragestellungen der Arbeitsgestaltung wird anhand aktueller Beispiele beleuchtet. Diese reichen von psychischen Belastungen, Zuverlässigkeit bis hin zur Gestaltung multimodaler Rückmeldesysteme.

Dieser Beitrag geht zunächst auf das Phänomen und die Bedeutung der Kompatibilität sowie auf das Wickens-Schema als modellhafte Beschreibung und die physiologischen Grundlagen der Kompatibilität ein. Die Bedeutung der Kompatibilität wird anhand von Studien aus der Montage und Fertigungsindustrie, dem Flugbereich, dem Einsatz digitaler Informationssysteme in der Aus- und Weiterbildung und der Gestaltung multimodaler Schnittstellen aufgezeigt. Abschließend wird die Bedeutung des Konstruktes für eine moderne Arbeitsgestaltung hinsichtlich psychischer Gesundheit diskutiert und Kompatibilität von psychischen Effekten der Monotonie und Vigilanz abgegrenzt.

Praktische Relevanz: Kompatibilität ist eines der zentralen arbeitswissenschaftlichen Konstrukte. Es wird seit Anbeginn der ergonomischen Arbeitsgestaltung untersucht und immer wieder stellt sich die Frage, inwieweit das Konstrukt Kompatibilität hinreichend erforscht und in der Praxis umgesetzt ist. Der Beitrag zeigt, dass Kompatibilität keinesfalls ein veraltetes Konstrukt ist, sondern durch neuere - auch physiologische Erkenntnisse - für die moderne Arbeitsgestaltung insbesondere im Rahmen der Digitalisierung ein essenzielles Konstrukt darstellt. Dies wird am Beispiel dreier Gestaltungsprobleme hochmoderner Arbeitssysteme aufgezeigt.

Schlüsselwörter Kompatibilität · Blickbewegung Analysen · Psychische Belastungen · Menschliche Zuverlässigkeit · Mensch-Roboter-Kollaboration · Head-Mounted Displays · Autonomes Fahren

Prof. Dr. phil. habil. Oliver Sträter

straeter@uni-kassel.de

1 Arbeits- und Organisationspsychologie, Universität Kassel,

Heinrich-Plett-Straße 40, 34131 Kassel, Deutschland 


\title{
Significance of compatibility for a safe and stress-free work design-Current relevance of the construct using the example of modern work design
}

\begin{abstract}
Compatibility has been one of the central concepts of work design since the very beginning of ergonomic research. Even if, or precisely because, the concept of compatibility is established, the importance of compatibility for current work design issues often takes a back seat because it is an old and therefore supposedly antiquated concept. The very fact that compatibility is essentially physiological, i.e. conditioned by our brain anatomy, shows that exactly the opposite is the case. The importance of compatibility for current work design issues is highlighted using current examples. These range from mental stress and reliability to the design of multimodal feedback systems.

This article deals first with the phenomenon and the importance of compatibility as well as the Wickens-scheme as a model description of the physiological basis of compatibility. The importance of compatibility is shown based on studies from the assembly and manufacturing industry, the flight sector, the use of digital information systems in training and further education and the design of multimodal interfaces. Finally, the meaning of the construct for a modern work design with regard to mental health is discussed and the compatibility of mental effects of monotony and vigilance is differentiated.

Practical Relevance: Compatibility is one of the central ergonomic constructs. It has been investigated since the beginning of ergonomic work-design and the question arises repeatedly to what extent the construct of compatibility has been adequately researched and implemented in practice. The article shows that compatibility is by no means an outdated construct, due to more recent—including physiological findings—it shows to be an essential construct for modern work design, especially in the context of digitalization, and demonstrates this using examples of three design problems of ultra-modern work systems.
\end{abstract}

Keywords Compatibility $\cdot$ Eye movement analyzes $\cdot$ Mental stress $\cdot$ Human reliability $\cdot$ Human-robot collaboration $\cdot$ Head-mounted displays $\cdot$ Autonomous driving

\section{Theorie und Bedeutung der Kompatibilität}

\subsection{Ausgangsbetrachtungen}

Kompatibilität ist aus gutem Grund seit den frühen Anfängen arbeitswissenschaftlicher Forschung eines der zentralen Konzepte der Arbeitsgestaltung. In seiner ursprünglichen Wortbedeutung bezeichnet Kompatibilität die Fähigkeit zweier Systeme, störungsfrei zusammenzuarbeiten zu können, ohne dass Anpassungen zwischen den Systemen erforderlich sind (Bubb 2015, S. 269; vgl. auch Wikipedia 2021). Dies gilt für technische, biologische oder kybernetische Fragestellungen gleichermaßen und folglich auch für arbeitswissenschaftliche Betrachtungen. Betrachtet man das Arbeitssystem des Menschen, geht es bei der Kompatibilität der Definition folgend um das störungsfreie Zusammenarbeiten des Menschen mit dem Arbeitssystem.

Die Kompatibilitätsforschung wird in diesem allgemeinen Kontext oft als Forschungskonstrukt für ganz spezifische Aspekte des Arbeitssystems verstanden, wie bei der Anordnung von Stellteilen/Bedienelementen oder Anzeigen, wobei das Arbeitssystem üblicherweise mithilfe des Mensch-Maschine-Systems beschrieben wird. Die Definition zeigt aber, dass der Begriff der Kompatibilität bei weitem nicht nur auf diese Aspekte des Arbeitssystems bezogen ist. In seiner allgemeinen Fassung bezeichnet Kompatibilität eher eine ganz zentrale arbeitswissenschaftliche Forderung an die Arbeitsgestaltung im Allgemeinen, dass der Mensch in seinem Arbeitssystem störungsfrei und optimal agieren kann (vgl. Strasser 1993, 2008). Gelingt keine störungsfreie Gestaltung, ist der Mensch gezwungen, Anpassungsleistungen zu erbringen. Bubb (1993) bezeichnet diese Anpassungsleistungen als Umkodierungsaufwand, da der Mensch die ihm dargebotene Information in die von ihm eigentlich erforderliche Information umwandeln muss.

Neben dem oft mit dem Begriff Kompatibilität in $\mathrm{Zu}-$ sammenhang gebrachten Verständnis, dass es um Kompatibilität von Stellteilen und Anzeigen geht, kann sich Kompatibilität durchaus auch auf ganz andere Aspekte des Arbeitssystems beziehen, wie beispielsweise Kompatibilität zwischen Führungskraft und Mitarbeitenden, Kompatibilität zwischen Management-Prozessen und Arbeitsmitteln o. ä. Damit zeigt der Begriff der Kompatibilität einen Forschungs- und Gestaltungsansatz auf, der für viele aktuelle Fragestellungen in der Arbeitsgestaltung von Bedeutung ist und ein allgemeines Konzept für die Arbeitsgestaltung darstellt.

Aus der Etymologie des Begriffes ergibt sich ferner, dass für eine gute Arbeitsgestaltung die Eigenschaften und Fähigkeiten des Menschen bekannt sein müssen. Der Gestaltungsansatz der Kompatibilität ist also ein vom Menschen ausgehender Gestaltungsansatz, der durch Kenntnis der Eigenschaften und Fähigkeiten des Menschen ein technisches System oder Arbeitsprozesse gestaltet. Damit ist Kompatibilitätsforschung ein proaktiver Gestaltungsansatz, welcher für Themen wie Digitalisierung, autonomes Fahren oder 
Mensch-Roboter-Kollaboration ganz entscheidende Impulse liefert. Im Gegensatz dazu wird allzu oft im Rahmen der Arbeitsgestaltung das technische System und seine Möglichkeiten vorangestellt und der Mensch als adaptives Element des Arbeitssystems den Eigenschaften der Technik angepasst. Dies führt zu einer zunehmenden Entgrenzung des Menschen (Sträter 2021) oder, wie Sheridan (2002) es ausdrückt, zu einer „Versklavung“ des Menschen durch die Technik. Kompatibilität als Gestaltungsansatz wirkt dieser Entwicklung entgegen und ist damit eine zentrale Anforderung an Arbeitsgestaltung im Rahmen von Digitalisierung (Sträter und Bengler 2019).

Ausgangspunkt dafür, das Konstrukt der Kompatibilität auf komplexe Arbeitssysteme anzuwenden, ist ein vertieftes Verständnis der aus der Kompatibilitätsforschung hervorgegangenen Erkenntnisse hinsichtlich menschlicher Eigenschaften und Fähigkeiten. Diese können auf unterschiedlichen Ebenen beschrieben werden (Sträter und Bubb 2003):

Jeder Mensch führt jegliche Aufgabenstellung und Tätigkeit mit einer aus seiner Erfahrung entwickelten Modellvorstellung über die Funktionsweise des Arbeitssystems durch (Bubb 1993). Diese Modellvorstellung wird üblicherweise als mentales Modell des Nutzers bezeichnet (Gentner und Stevens 1983). Kompatibilität auf dieser Ebene der Betrach- tung bezeichnet, inwieweit das individuelle mentale Modell des Menschen mit den Funktionen sowie den erforderlichen Aufgaben und Tätigkeiten innerhalb des gegebenen Arbeitssystems zusammenpasst (sogenannte primäre interne Kompatibilität; Bubb 2015, S. 269). Hier geht es um die Passung des mentalen Modells mit der Realität des Arbeitssystems (je weniger das mentale Modell zu den Funktionen, Aufgaben und Tätigkeiten passt, desto höher ist die zu erbringende Anpassungsleistung). Diese Anpassungsleistung unterteilt sich weiter: Sie kann räumlich gesehen werden, sich also auf Anordnungen von Bediensystemen für unterschiedliche Aufgaben und Tätigkeiten beziehen oder zeitlich, sich also auf eine Vergleichbarkeit von Vorgängen für unterschiedliche Aufgaben und Tätigkeiten beziehen.

Daneben gibt es, insbesondere in komplexen Arbeitssystemen, immer eine zweite erforderliche Anpassungsleistung des Menschen, die darin besteht, dass der Mensch im Arbeitssystem jeweils unterschiedliche Informationen aufzunehmen oder unterschiedliche Aufgaben und Tätigkeiten durchzuführen hat. Die hier erforderliche Anpassungsleistung ergibt sich aus der Passung der unterschiedlichen Aufgaben und Tätigkeiten untereinander (sogenannte primäre externe Kompatibilität; Bubb 2015). Hier geht es also um den Umkodierungsaufwand bei der Bearbeitung unter-

Tab. 1 Kompatibilitätsarten mit Beispielen und Zusammenhang zur Interferenz im Sinne des Wickens-Schemas Table 1 Compatibility types with examples and connection to interference in the sense of the Wickens-scheme

\begin{tabular}{llll}
\hline $\begin{array}{l}\text { Kompati- } \\
\text { bilität }\end{array}$ & Negativ-Beispiel & Inkompatibilität & \multicolumn{1}{l}{$\begin{array}{l}\text { Interferenz im Sinne des Wi- } \\
\text { ckens-Schemas }\end{array}$} \\
\hline $\begin{array}{l}\text { Primäre interne Kompatibilität } \\
\text { Räumlich }\end{array}$ & $\begin{array}{l}\text { Eine Bewegung der Maus nach vorn bewegt den } \\
\text { Zeiger auf dem Bildschirm nach unten }\end{array}$ & $\begin{array}{l}\text { Die Zuordnung der räumlichen Ko- } \\
\text { dierung steht im Widerspruch zu den } \\
\text { räumlich organisierten Gedächtnisein- }\end{array}$ & $\begin{array}{l}\text { Interferenz durch Widerspruch } \\
\text { zwischen räumlichen Gedächt- } \\
\text { nisinhalten und räumlicher }\end{array}$ \\
Zeitlich & $\begin{array}{l}\text { Zeiten } \\
\text { Zeitliche Abläufe (Prozess-, Flussdiagramme) wer- } \\
\text { den von rechts nach links oder von unten nach oben } \\
\text { angeordnet }\end{array}$ & $\begin{array}{l}\text { Die Zuordnung der zeitlichen Kodie- } \\
\text { rung steht im Widerspruch zu den zeit- } \\
\text { lich organisierten Gedächtnisinhalten }\end{array}$ & $\begin{array}{l}\text { Interferenz durch Widerspruch } \\
\text { zwischen zeitlichen Gedächt- } \\
\text { nisinhalten und zeitlicher Ko- } \\
\text { dierung }\end{array}$
\end{tabular}

Primäre externe Kompatibilität

Räumlich Die rechte Cursortaste bewegt den Cursor nach links

Die räumliche Zuordnung von Ort der Tätigkeit und Ort des Cursors stimmen nicht überein

Zeitlich Die Sprachausgabe eines Navigationssystems erfolgt zeitlich nach Auftauchen der Straßenführung im Blickfeld und die erforderliche Handlung wird nicht rechtzeitig eingeleitet

Sekundäre Kompatibilität

Uni- Hängender Zeiger mit Linksdrehung und einer

modal Skalierung von links gleich wenig zu rechts gleich viel

Multi- Bei einer Parkhilfe wird der Gefahrenbereich vorn modal rechts angezeigt, der Alarm-Ton kommt für den Fahrer aus dem linken Lautsprecher
Die zeitliche Blickführung und das auditive Signal passen nicht überein

Die räumliche Anordnung von wenig zu viel (räumlich) widerspricht der Bedeutung von weniger bei Linksdrehung des Zeigers (zeitlich)

Widerspruch zwischen räumlicher Anordnung auf dem Display (visuelle) und akustischer Rückmeldung des Lautsprechers (auditive)
Interferenz in der räumlichen Kodierung bezüglich der Anordnung der Taste und Anordnung des Cursors

Interferenz in der zeitlichen Kodierung

Interferenz zwischen räumlicher und zeitlicher Kodierung innerhalb einer Modalität

Interferenz zwischen visueller und auditiver Modalität innerhalb der räumlichen Kodierung 
schiedlicher Aufgaben oder einer Aufgabensequenz (je heterogener die unterschiedlichen Aufgaben und Tätigkeiten, desto höher ist die erforderliche Anpassungsleistung).

Weiterhin ergeben sich Probleme der Kompatibilität, wenn ein innerer Widerspruch zwischen Teilaspekten einer Aufgabe oder Tätigkeit vorhanden ist. Dieser Widerspruch kann in einer unimodalen Informationsübermittlung durch inkompatible Informationsdarstellung einer räumlichen oder zeitlichen Kodierung zustande kommen oder bei einer multimodalen Informationsübermittlung zusätzlich durch eine inkompatible Informationsdarstellung über unterschiedliche Sinnesmodalitäten hinweg (sogenannte sekundäre Kompatibilität; Bubb 2015).

Beispiele für diese unterschiedlichen Arten der Kompatibilität sind in Tab. 1 aufgeführt. Inkompatible Gestaltungslösungen erfordern Anpassungsleistungen des Menschen und damit immer einen kognitiven Aufwand, die Widersprüche in der Darstellung aufzulösen. Um das Konstrukt der Kompatibilität als proaktives Gestaltungskonstrukt einzusetzen, ist somit ein Verständnis der Eigenschaften der menschlichen Informationsverarbeitung von Bedeutung. Im Folgenden werden deshalb wesentliche Zusammenhänge von Kompatibilität, kognitiven Eigenschaften und ergonomischen Anforderungen dargestellt. Es folgen Beispiele der Bedeutung von Kompatibilität für die Gestaltung moderner Arbeitssysteme.

\subsection{Zusammenhänge - Kompatibilität, Ressourcen- Theorie, Physiologie und Systemergonomie}

Das Faktum, dass Inkompatibilitäten zu kognitiven Aufwänden führen zeigt, dass ein Verständnis über die Bedeutung von Kompatibilität mit einem Verständnis über die Eigenschaften und Fähigkeiten der menschlichen Informationsverarbeitung einhergeht (Bubb und Sträter 2006). Eine eng mit der Kompatibilitätsforschung zusammenhängende Modellierung dieser kognitiven Aufwände hat Wickens (1984) zu einem Schema zusammengefasst, welches wesentliche kognitive Eigenschaften des Menschen bei der Interaktion mit einem Arbeitssystem zusammenfasst, dem sogenannten Wickens-Schema. Er unterscheidet dabei im Wesentlichen drei Komponenten, die für die Informationsverarbeitung und für dabei entstehende Kompatibilitätsprobleme zuständig sind. Dies sind zunächst einmal die Kodierung der zu verarbeitenden Informationen (in räumlich und zeitlich), die Sinneskanäle (Modalitäten) sowie unsere Gedächtnisinhalte (in räumlich und zeitlich).

Da sich Wickens hauptsächlich mit audiovisuellen Arbeitsumgebungen beschäftigt hat, unterscheidet das Ursprungsmodell, bezogen auf die Sinneskanäle, zwei Modalitäten (auditiv und visuell). Burghardt (2019) konnte am Beispiel der taktilen Informationsaufnahme und -verarbeitung zeigen, dass die von Wickens gefundenen $\mathrm{Zu}$ - sammenhänge auch auf andere Modalitäten übertragbar sind. Neuere Forschungen im Bereich der Neuro-Psychologie zeigen zusätzlich, dass die im Wickens-Schema repräsentierten Zusammenhänge einen eindeutigen Bezug zur Physiologie des Gehirns und zum kognitiven Verarbeitungszyklus des Menschen haben (Sträter 2005) und die von Wickens identifizierten Komponenten eindeutig neuroanatomisch zugeordnet werden können. Kodierung und Modalitäten sind Strukturmerkmale der Gyrus Cinguli, einer Hirn-Struktur, die sich unmittelbar an das limbische System anschließt und für die räumliche und zeitliche Kodierung der über die Sinnesmodalitäten aufgenommenen Informationen zuständig ist. Die Struktur des Gyrus Cinguli dient also der ersten inhaltlichen Aufbereitung der von außen vom Menschen aufgenommenen Information. Der Gyrus Cinguli ist mit der linken und rechten Hemisphäre über Pyramidenzellen verbunden. Die eigentlichen Gedächtnisinhalte sind im kortikalen System der Hemisphären abgelegt. Die im Gyrus Cinguli raum/zeitlich organisierten Informationen werden in einem kognitiven Verarbeitungszyklus mit den in den Hemisphären abgelegten Gedächtnisinhalten abgeglichen. Dieser AbgleichsProzess wird von der Alphafrequenz getriggert und erfolgt in ca. $100 \mathrm{~ms}$ pro Abgleich, wobei sich diese Zeit altersabhängig zwischen ca. $70 \mathrm{~ms}$ bei Neugeborenen und ca. $130 \mathrm{~ms}$ bei 90 -jährigen verändert.

Das sich ergebende erweiterte Wickens-Schema ist mit den physiologischen Zuordnungen in Abb. 1 zusammengefasst. Wickens konnte dabei zeigen, dass es immer dann Kompatibilitätsprobleme gibt, wenn die Zuordnung zu der in den Gyrus Cinguli vorstrukturierten Information nicht eindeutig den Gedächtnisinhalten zuordbar ist. Wickens bezeichnet diese Zuordnungsprobleme als Interferenz, denn die Probleme der Zuordnung kommen durch die Überlagerung der unterschiedlichen Inhalte von Gyrus Cinguli und Hemisphäre zustande. Physiologisch ist diese Zuordnung durch den Verarbeitungszyklus des Menschen begründet (Sträter 2005). Innerhalb eines Verarbeitungszyklus des Menschen wird eine ,dynamische Bindung (eine stabile Aktivierung) zwischen Gyrus Cinguli und Kortex aufgebaut, welche die inhaltliche Zuordnung und Interpretation der im Gyrus Cinguli abgelegten Aktivierungen repräsentiert. Hierdurch ist der Mensch in der Lage, einen Sachverhalt, welcher in seinem raumzeitlichen Umfeld über die Sinnesmodalitäten erfahren wird, inhaltlich zu erkennen.

Interferenz, also das nicht effiziente Verarbeiten von unterschiedlichen Informationen, kommt immer dann zustande, wenn Inkompatibilitäten in oder zwischen Kodierung und Modalität herrschen, denn diese resultieren in eine mehrdeutige Zuordnung der Kodierung zu den Gedächtnisinhalten. Als logische Konsequenz treten Interferenzen laut Wickens immer dann auf, wenn unterschiedliche Informationen von ein und derselben Ressource zu verarbeiten sind; 
Abb. 1 Wickens-Schema erweitert um unterschiedliche Modalitäten und mit physiologischen Zuordnungen. (Aufbauend auf Wickens 1984; Burghardt 2019; Sträter 2005)

Fig. 1 Wickens-scheme expanded by different modalities and with physiological assignments. (Based on Wickens 1984; Burghardt 2019; Sträter 2005)

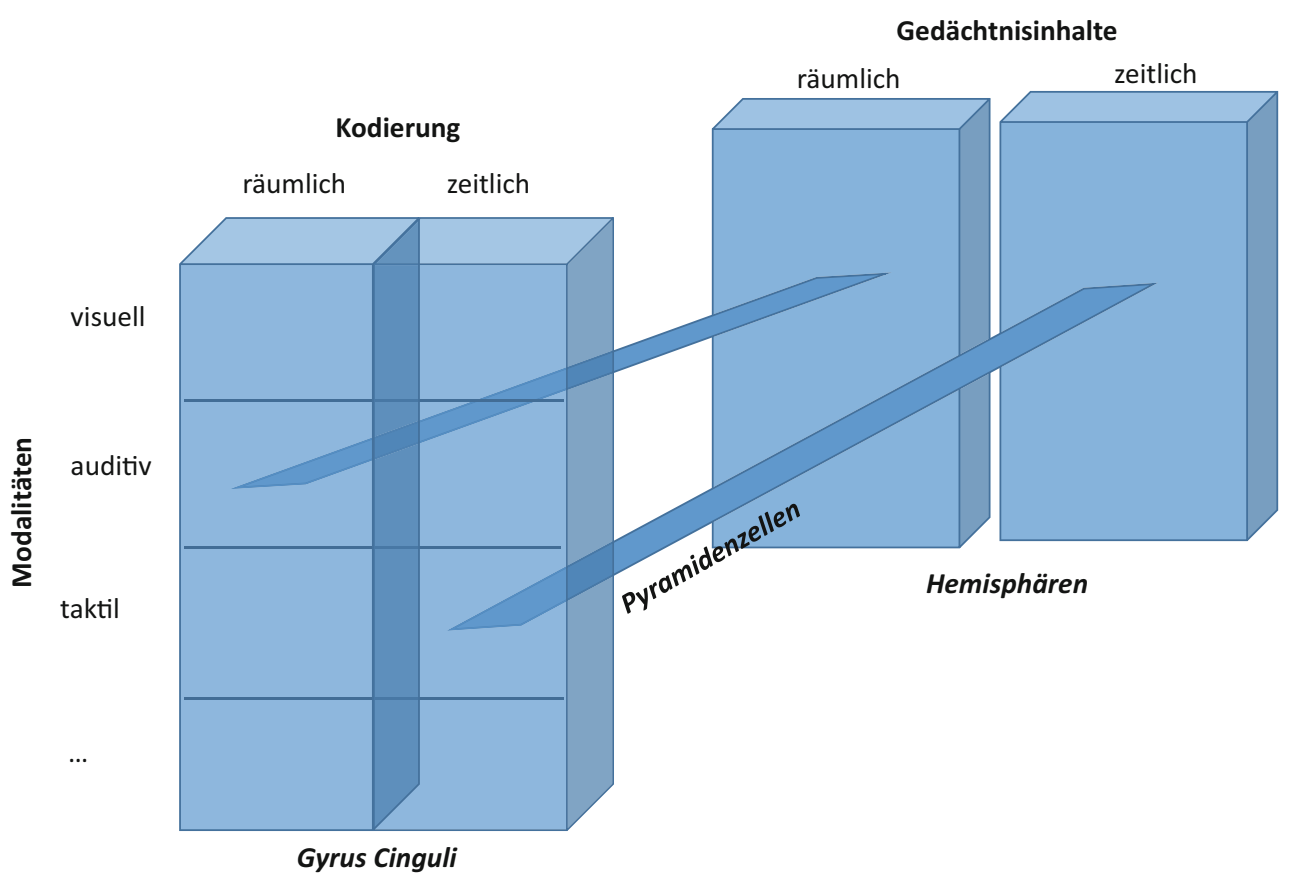

das Wickens-Schema wird deshalb kognitionswissenschaftlich auch als Theorie der multiplen Ressourcen bezeichnet (Low 2003).

Mithilfe der multiplen Ressourcentheorie lassen sich die oben angesprochenen Kompatibilitätsproblematiken herleiten und weiter strukturieren. Inkompatibilitäten entstehen immer dann,

- wenn unterschiedliche Informationen mit demselben Format und derselben Modalität einem Gedächtnisinhalt zugeordnet werden müssen (Interferenz innerhalb eines Formates und einer Modalität)

- wenn unterschiedliche Informationen in demselben Format, aber unterschiedlichen Modalitäten übermittelt wird (Interferenz innerhalb eines Formates)

- wenn über eine gleiche Modalität unterschiedliche räumliche bzw. zeitliche Information übermittelt wird (Interferenz innerhalb der Modalität)

Interferenzen beschreiben den Basiseffekt der Kompatibilität. Diese Basisprozesse sind in Tab. 1 mit typischen Beispielen von Kompatibilitätsproblemen aufgeführt.

Interferenzen können im Umkehrschluss immer dann vermieden werden, wenn unterschiedliche Informationen in einem unterschiedlichen Format und in unterschiedlicher Modalität übermittelt werden. Ergonomisch optimal ist es, wenn sich die Informationen innerhalb einer Modalität inhaltlich ergänzen und kongruent in den Formaten kodiert sind. In diesem Fall ergänzen sich die Zuordnungen der beiden Hemisphären gegenseitig und es ergibt sich ein geschlossenes und stimmiges Bild für den Nutzer, welches als intuitiv richtig empfunden wird.
Herrschen Interferenzen vor, so setzt dies immer voraus, dass eine inkompatible Darstellung der Information vorliegt (vgl. Spanner 1993). Der Umkodierungsaufwand liegt darin, dass in diesem Fall die Interferenz aufgelöst werden muss, indem die Modalitäten oder Kodierungen getrennt und nacheinander verarbeitet und den Gedächtnisinhalten zugeordnet werden. Hierzu ist eine getrennte Fokussierung auf die einzelnen Informationseinheiten erforderlich, und damit wird Aufmerksamkeit entsprechend gebunden (gerichtete Aufmerksamkeit). Ferner sind für den Abgleich kognitive Verarbeitungszyklen erforderlich, und damit ist der gesamte kognitive Apparat mit der Umkodierung beschäftigt, kann also keine weiteren Verarbeitungsprozesse durchführen.

\subsection{Bedeutung einer kompatiblen Gestaltung für die Reduktion kognitiver Interferenz}

Die enge Beziehung von Kompatibilität mit der multiplen Ressourcentheorie sowie deren eindeutiger Bezug zu physiologischen Sachverhalten zeigt, dass die Forderung nach Kompatibilität keine, weiche ' Forderung an eine Arbeitsgestaltung sein kann. Kompatibilität ist in den Hirnstrukturen verankert, welche weder durch Training noch durch Lernen veränderbar sind. Kompatibilitätsprobleme führen deshalb zu einem dauerhaften kognitiven Aufwand der Umkodierung und sind keine sich irgendwann auflösende bzw. durch Lernen zu kompensierende Gestaltungsdefizite.

Vielmehr zeigt dieser eindeutige Zusammenhang, dass nur durch eine Arbeitsgestaltung, die die Prinzipien der Kompatibilität berücksichtigt, mentale menschliche Eigen- 
schaften in der Informationsverarbeitung berücksichtigt werden können. Für einen proaktiven Gestaltungsansatz hinsichtlich mentaler Belastungsfaktoren für die menschliche Arbeit stellt die Kompatibilität also eine Schlüsselgröße dar (siehe hierzu auch Strasser 2021).

Gerade in der Phase hoher Digitalisierung, in der viele Inkompatibilitäten aufgrund der Heterogenität der Systeme entstehen, ist deshalb eine durchgängige Berücksichtigung dieses Gestaltungsansatzes von entscheidender Bedeutung. Die Bedeutung der Kompatibilität für aktuelle Fragestellungen der Arbeitsgestaltung wird anhand dreier Beispiele aus den Bereichen menschliche Zuverlässigkeit, psychische Belastung und Gestaltung multimodaler Rückmeldesysteme beleuchtet.

\section{Empirische Studien zur Bedeutung der Kompatibilität}

\subsection{Cockpitgestaltung in der Luftfahrt}

In der Luftfahrt ist die Bedeutung der Kompatibilität seit Anbeginn der Human-Factors-Forschung ein wichtiges Auslegungskriterium. Der Grund liegt darin, dass in der Luftfahrt in kritischen Situationen hochdynamische und zeitkritische Tätigkeiten von den Pilot:innen erforderlich werden. Wie oben beschrieben führen Inkompatibilitäten dazu, dass mindestens ein weiterer kognitiver Verarbeitungszyklus erforderlich wird. Damit ist das kognitive System der Pilot:innen mit Auflösung der Inkompatibilität beschäftigt und für die eigentliche Flugregelaufgabe geht Zeit verloren. Der Arbeitsplatz einer Pilot:in ist deshalb hinsichtlich des Aspektes der Kompatibilität üblicherweise gut ausgelegt.

Dennoch zeigen sich immer wieder Gestaltungsprobleme in der Cockpitgestaltung, die auf Kompatibilitätsprobleme zurückzuführen sind. In einer Studie des Flugunternehmens TUI wurden Leistungsunterschiede von Pilot:innen bei Überprüfungsflügen im Simulator in kritischen Situationen untersucht (Günebak et al. 2010). Anlass der Untersuchung war, dass Unsicherheiten der Pilot:innen beim Handling mit dem sicherheitskritischen Szenario Triebwerksausfall in der Startphase beobachtet wurden und sich weder Pilot:innen noch Überprüfungspilot:in den Grund für diese Unsicherheiten erklären konnten. Es wurde eine Untersuchung aufgesetzt, bei der Blickbewegungsmessungen der Pilot:innen beim Triebwerksausfall im Simulator durchgeführt wurden, um den Grund für die Unsicherheiten zu eruieren.

Abb. 2 zeigt zunächst das Szenario, wie es sich aus Sicht der Pilot:innen darstellt. Ein Triebwerksausfall einer Passagiermaschine mit zwei Turbinen äußert sich darin, dass ein heftiger Drehimpuls auf die Maschine ausgeübt wird. Bei Ausfall des linken Triebwerks wird ausschließlich Schub des rechten Triebwerks geleistet, und das Flugzeug erhält einen Drehimpuls nach links. In einem Steigflug, wie er in der Startphase erforderlich ist, geht dieser Drehimpuls auch in eine zusätzliche Steigrate des Flugzeugs über und damit in die Gefahr des Strömungsabrisses. Die Pilot:innen müssen in der ersten Phase des Ausfalls das Flugzeug zunächst stabilisieren und das defekte Triebwerk so schnell wie möglich aufgrund von möglichen Folgeschäden (Brand, Explosion) abschalten. Entscheidend für beide Aufgaben sind Fluglage und Diagnose, welches Triebwerk geschädigt ist. Die Abschaltung des falschen Triebwerks hätte un-
Abb. 2 Darstellung des Triebwerksausfalls im Cockpit eines Passagierflugzeugs (die Pfeile stellen die jeweiligen Bewegungsrichtungen bei Triebwerksausfall des linken Triebwerks dar)

Fig. 2 Representation of the engine failure in the cockpit of a passenger aircraft (the respective directions of movement in the event of engine failure of the left engine are marked with arrows)

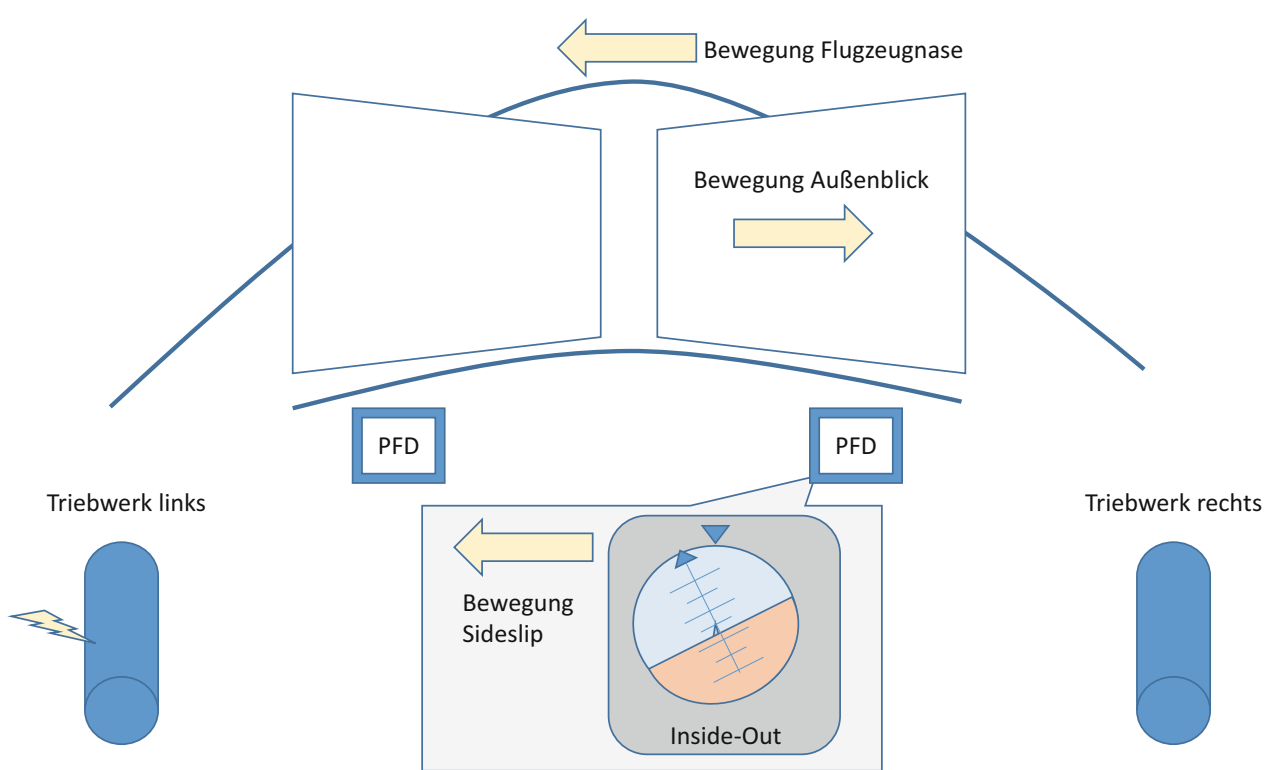


mittelbar den Totalverlust der Maschine zur Folge, wie es beispielsweise bei dem Unfall von British Midland 1989 geschehen ist (Airsafe 2002).

Sieht die Pilot:in beim Triebwerksausfall aus dem Cockpit-Fenster, wird der Triebwerksausfall durch Verschiebung des Wolkenbildes nach rechts erkannt. Nach dem Prinzip der primären externen Kompatibilität ist hierdurch ersichtlich, dass das rechte Triebwerk mehr Schub hat. Blickt die Pilot:in beim Triebwerksausfall auf das primäre Flugdisplay (Primary Flight Display - PFD), wird der Triebwerksausfall an einer links-Verschiebung des sogenannten Side-Slip-Indikators erkannt. Diese Bewegung entspricht der Bewegung der Flugzeugnase und ist nach dem Prinzip des sogenannten Inside-Out kompatibel zu dieser gestaltet: das Display zeigt den künstlichen Horizont aus Sicht des Cockpits so, wie die Pilot:in es auch sieht. Von der Kompatibilität her ist dies die richtige Gestaltung für den Flugbetrieb nach dem Prinzip der sekundären Kompatibilität. Sie ist für den normalen Flugbetrieb auch sinnvoll, denn bei Nebel oder Flug durch Wolken entfällt der Außenblick und die Pilot:innen haben durch diese Gestaltungslösung die Möglichkeit, einen Quasi-Außenblick mit künstlichen Horizont zu erhalten.

Die Darstellung des Slide-Slip-Indikators steht jedoch im Widerspruch zur primären externen Kompatibilität. Wenn also sowohl Außenblick als auch Side-Slip-Indikator zur Verfügung stehen und ein Triebwerksausfall vorliegt, sind widersprüchliche Bewegungsrichtungen $\mathrm{zu}$ interpretieren. Dass diese Inkompatibilität bedeutend für die Leistungsfähigkeit von Pilot:innen im Szenario Triebwerksausfall ist, wurde von Günebak et al. (2010) gezeigt: Pilot:innen, die während des Triebwerksausfalls ihren Blick auf die Flugzeugumgebung gerichtet haben (Außenblick), konnten wesentlich schneller und zuverlässiger sowohl das defekte Triebwerk identifizieren als auch das Flugzeug stabilisie- ren. Pilot:innen, die während des Ausfalls des Triebwerks auf das Primary Flight Display blickten, wiesen dagegen Unsicherheiten sowohl in der Stabilisierung des Flugzeugs als auch in der Zuordnung des defekten Triebwerks auf. Diese Unsicherheiten äußerten sich in einer reduzierten Regelgüte und längeren Zeiten bei der Durchführung der erforderlichen Tätigkeiten. Der Grund kann eindeutig der Kompatibilitätsproblematik zugeordnet werden: Pilot:innen, die auf das Primary Flight Display schauen, sehen den Side-Slip-Indikator nach links verschieben und müssen ihr mentales Modell nutzen, um diese Bewegungsrichtung mit dem ungleichen Schub der Triebwerke in Einklang zu bringen, denn das mentale Modell (also die primäre externe Kompatibilität) erwartet, dass sich etwas nach rechts bewegt, wenn auf der rechten Seite mehr Schub gegeben wird. Pilot:innen, die nach außen schauen, haben diesen Umkodierungsaufwand nicht zu leisten. Die in der Untersuchung gefundenen Ergebnisse wurden aufgrund ihrer Bedeutung seitens TUI unmittelbar in das Training beim Umgang mit Triebwerksausfällen integriert.

\subsection{Kompatibilität zwischen Arbeitsstationen in der Montage und Fertigung}

Die Fertigungs- und Montageindustrie ist durch hohe Produktivität bei gleichzeitig hoher Sicherstellung der Gesundheit der Mitarbeitenden charakterisiert. Sowohl für Produktivität als auch für Gesundheit sind inkompatible Arbeitssituationen ungünstig, denn sie erhöhen den mentalen Aufwand der Mitarbeitenden und damit die psychische Belastung. Gleichermaßen reduzieren sie die Produktivität, da die erforderlichen Verarbeitungszyklen zur Umkodierung nicht durch Erfahrung oder Training kompensierbar sind. In unterschiedlichen Studien in der Fertigungs- und Montage-
Abb. 3 Das Mess-System cEYEberman mit Bewegungsund Blickbewegung-Aufnahme sowie Möglichkeiten der Analyse und Bewertung

Fig. 3 The measuring system cEYEberman with movement and eye movement recording as well as possibilities for analysis and evaluation

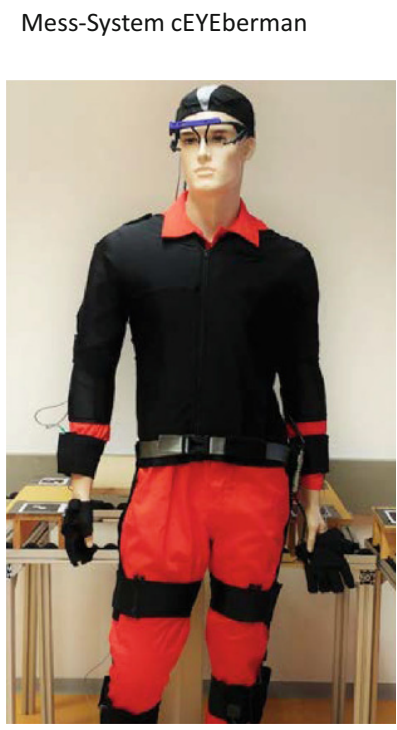

Synchrone Datenerfassung von Blick- und Bewegungsdaten
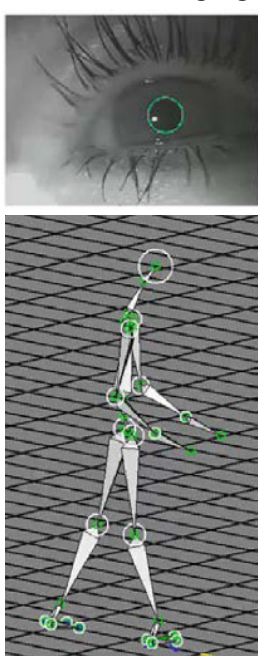

Ergonomische

Analyse und Bewertung

Analyse und Bewertung mit Hilfe von bekannten ergonomischen Grenzwerten oder KI-Verfahren von:

- Menschlicher Zuverlässigkeit Ergonomische Gestaltung von Cockpits oder Bildschirmsystemen

- Arbeitssystemgestaltung bei multiplen Arbeitsstationen Kombinierte psychische und physische Belastung über Blick- und Bewegungsanalyse Verhaltensanalyse und training 
industrie wurden Fertigungs- und Montagelinien hinsichtlich möglicher psychischer und physischer Arbeitsbelastungen untersucht (Sträter et al. 2018; Metternich und Sträter 2020). Dabei wurde das Blick- und Bewegungsmesssystem ,cEYEberman' eingesetzt, welches über Bewegungssensoren (Gyroskope) und eine Blickbewegungsbrille sowohl das Bewegungsverhalten als auch das Blickverhalten einer arbeitenden Person zeitsynchron aufnehmen kann und diese hinsichtlich ergonomischer Kriterien sowie psychischer und physischer Belastungen auswerten kann (Klippert et al. 2011; Arenius et al. 2013). Abb. 3 zeigt das System und die Stufen der Auswertung.

Mithilfe des Mess-Systems können Arbeitsbelastungen sehr effizient untersucht und Schwachstellen im Arbeitssystem ermittelt werden, da ergonomisch ungünstige Haltungen im Bewegungsapparat durch die über die Blickbewegung ersichtlichen Arbeitsinhalte erkannt werden und unmittelbar Schwachstellen in der ergonomischen Auslegung von Arbeitsplätzen identifiziert werden können. Das System wurde in vielfältigen Arbeitsplätzen der Fertigungsund Montageindustrie eingesetzt, um Belastungsfaktoren zu identifizieren. Immer wieder zeigten sich dabei - an sich offensichtliche - Kompatibilitätsprobleme, die gleichermaßen Probleme hinsichtlich der Produktivität und der Belastung der Mitarbeiter hervorrufen.

Ein Beispiel aus einer Arbeitsstation zur Platinen-Bestückung ist in Abb. 4a dargestellt. Sie zeigt, wie relevant eine nach Kompatibilitätsgrundsätzen gestaltete Arbeitsstation für Produktivität und Belastung der Mitarbeitenden ist, insbesondere hinsichtlich der räumlichen Kompatibilität zwischen Entnahmekästen und zu bestückender Platine. Hierzu ist in der Abb. 4b das Greif-Verhalten der rechten Hand zwischen Entnahmebehältern und zu bestückender Platine bei einer räumlich inkompatiblen Anordnung dargestellt. Diese besteht beispielsweise daraus, dass für das Bauteil unten links auf der Platine in den Entnahmebehälter oben rechts gegriffen werden muss. Durch Neuordnung der Entnahmebehälter in eine räumlich kompatible Anordnung ergibt sich das in Abb. 4c dargestellte Greif-Verhalten.
Wie aus der Abbildung ersichtlich, ist das Greif-Verhalten in der inkompatiblen Bedingung sehr heterogen. Dies sowohl hinsichtlich der Anzahl der Fixationen als auch hinsichtlich der Pfade. In der räumlich kompatiblen Bedingung (Entnahmebehälter rechts oben enthält Bauteile für den Platinenplatz rechts oben) ist das Greif-Verhalten sowohl zeitlich als auch räumlich augenfällig systematischer. Durch die Neuordnung wurde sowohl ein schnelleres Arbeiten als auch weniger Ermüdung der Mitarbeitenden festgestellt (Sträter et al. 2018).

Gerade bei flexibler Fertigung, bei der unterschiedliche Platinen an ein und derselben Arbeitsstation bestückt werden sollen, stellt sich aus ökonomischen Gründen immer die Frage, inwieweit es sich lohnt, den Arbeitsplatz bzw. die Entnahmebehälter auf die jeweilige Platine neu einzustellen. Die Ergebnisse der hier dargestellten Untersuchung zeigen aber, dass in jedem Fall eine nach Grundsätzen der Kompatibilität gestaltete Anordnung der Entnahmebehälter hinsichtlich der Faktoren Gesundheit und Produktivität lohnenswert ist.

Ähnliches zeigt sich beim Übergang zwischen unterschiedlichen Arbeitsstationen. In einer Untersuchung zu U-Linien konnte festgestellt werden, dass zwar einzelne Arbeitsstationen für sich ergonomisch gestaltet sind, jedoch nicht die Übergänge zwischen den Arbeitsstationen (Sträter et al. 2018). Dabei konnte mit Hilfe von Blickbewegungsuntersuchungen festgestellt werden, dass Inkompatibilität beim Übergang zwischen zwei Arbeitsstationen oft unnötige kognitive Beanspruchung der Mitarbeitenden mit sich bringt, gerade bei kurz-getakteten Arbeitsinhalten. Grund dafür ist, dass räumliche Kompatibilität zwischen Arbeitsstationen nicht gegeben ist. Sind beispielsweise im Entnahmebehälter an der Arbeitsstation A Schrauben oben rechts sortiert und in der folgenden Arbeitsstation B unten links, führt dies zu einer Inkompatibilität zwischen den Arbeitsstationen und damit immer wieder zu kognitiven Aufwänden, die mental belasten und ermüden sowie produktive Zeit kosten (Sträter et al. 2018).
Abb. 4 Die Reihenfolge der Fixationen bewegt sich von links oben bis rechts unten in $\mathbf{b}$ und von links nach rechts in $\mathbf{c}$ (Anfang der Greif-Sequenz bis Ende der Greif-Sequenz)

Fig. 4 The color scheme indicates the sequence of fixations from left-top to lower-right in $\mathbf{b}$ and left to right in $\mathbf{c}$ (beginning of the grasp-sequence to end of the grasp-sequence)

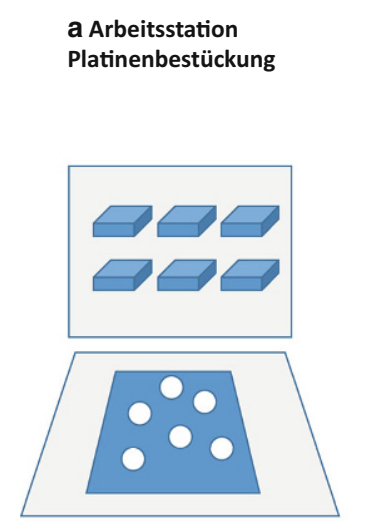

b Greif-Verhalten bei inkompatibler räumlicher Anordnung der Entnahmebehälter zur Bestückungsplatine

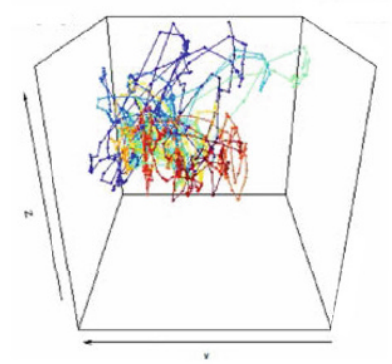

c Greif-Verhalten bei kompatibler räumlicher Anordnung der Entnahmebehälter zur Bestückungsplatine

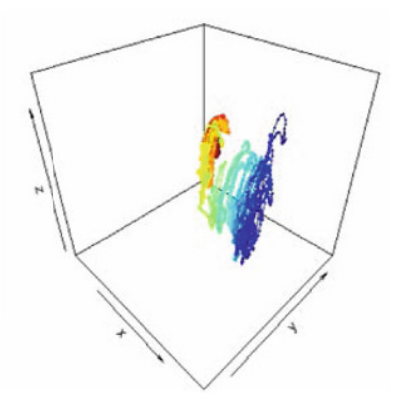




\subsection{Kompatibilität zwischen Modalitäten im Bereich von Assistenzsystemen}

Als drittes Beispiel wird auf die Bedeutung der Kompatibilität für multimodaler Schnittstellen eingegangen. Im Rahmen einer Untersuchung, inwieweit eine Echtzeit-Rückmeldung über die eigene unergonomische Haltung zu einer Verhaltensmodifikation der Mitarbeitenden führt, konnten Burghardt und Sträter (2017) eindeutig zeigen, dass neben dem Ursprungsmodell von Wickens sich auch andere Sinneskanäle, wie beispielsweise taktile Rückmeldung sinnvoll in das Interferenzschema integrieren lassen und Forschungsergebnisse damit eindeutig interpretierbar werden. Die Studie unterstreicht die Bedeutung der Kompatibilität für die Gestaltung von multimedialen Schnittstellen, wie sie derzeit im Rahmen der Digitalisierung in allen möglichen Arbeitsbereichen Einzug erhalten.

Burghardt (2019) untersuchte, inwieweit durch eine Echtzeit-Rückmeldung über ergonomische Haltungen das Verhalten von Mitarbeitenden hin zu einer verbesserten ergonomischen Haltung in der Fertigung unterstützt werden kann. Hierzu wurde der oben bereits angesprochene Messanzug cEYEberman eingesetzt und um eine Echtzeit-Rückmeldung der ergonomischen Haltung an den Mitarbeitenden ergänzt. Burghardt untersuchte dabei drei unterschiedliche Rückmeldesysteme. Diese sind in Abb. 5 dargestellt.

In der ersten Lösung wurde die visuelle Modalität mithilfe einer schematischen Darstellung des Muskel-SkelettSystems über eine Datenbrille an den Mitarbeitenden über-

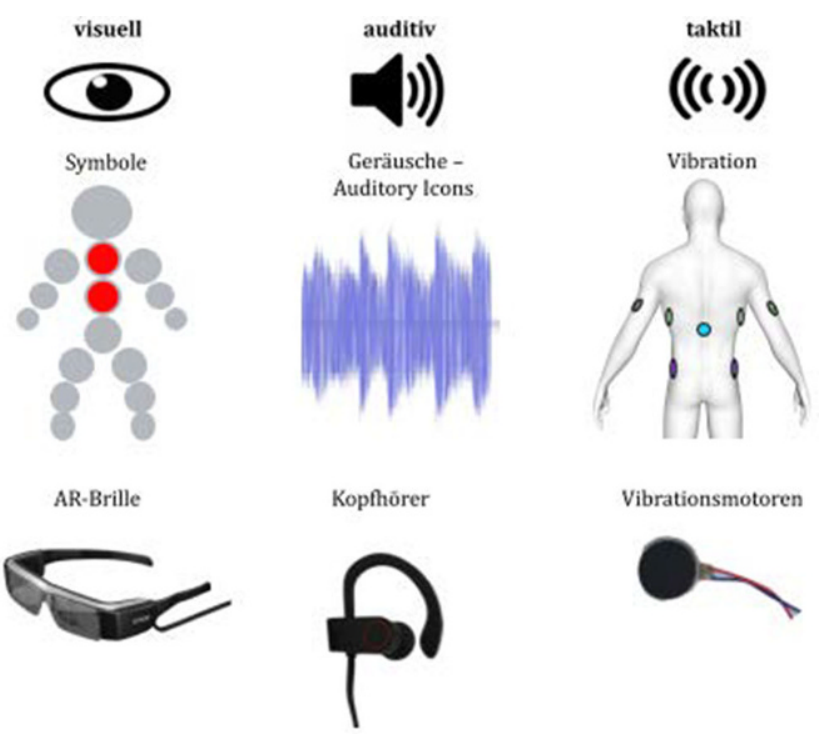

Abb. 5 Versuchsbedingungen unterschiedlicher Rückmeldesysteme zur Übermittlung nicht ergonomischer Haltungen. (Aus Burkhardt 2019, S. 143)

Fig. 5 Test conditions of different feedback systems for the transmission of non-ergonomic postures. (From Burkhardt 2019, S. 143) mittelt. Ergonomisch ungünstige Zustände wurden über rot gefärbte Teile des Körpers indiziert. In dieser Bedingung muss der Mitarbeitende die über die Datenbrille aufgenommene visuelle Information auf seinen eigenen Körper gedanklich projizieren. Hierzu muss die über die Datenbrille übermittelte räumlich visuelle Information (roter Kreis in der Mitte des Körpers) visuell räumlich erfasst werden. Die Haltungsindikation ist dabei im linken peripheren Bereich des Blickfeldes untergebracht, um die Hauptblickrichtung auf die Arbeitsinhalte nicht zu beeinträchtigen. Somit ist für die Übertragung der Information die Darstellung im linken Blickfeld zu erfassen und auf die zentrale Achse des Rückens gedanklich zu beziehen, um zu erkennen, dass es sich um eine unergonomische Haltung des eigenen Rückens handelt (räumliche Kodierung im Blickfeld ist auf räumliche Kodierung des Rückens zu projizieren). In der auditiven Bedingung wird diese Information über den auditiven Kanal vermittelt. Diese muss aufgenommen und gedanklich auf den eigenen Körper projiziert werden (zeitliche Kodierung der akustischen Information ist auf räumliche Kodierung des Rückens zu projizieren). In der taktilen Bedingung wird die ungünstige Haltung über Vibrationsmotoren an den entsprechenden Körperstellen übermittelt (räumliche Kodierung wird an genau der Stelle projiziert, die muskulär angesteuert werden muss; es ist keine Umkodierung erforderlich). Handelt es sich beispielsweise um eine ungünstige Rücken-Beugung, wird der entsprechende Vibrationsmotor an der Wirbelsäule aktiviert.

Burghardt (2019) konnte in seiner Studie eindeutig nachweisen, dass eine Rückmeldung, die direkt an der Stelle erfolgt, an der eine Verhaltensänderung erforderlich ist, die besten Ergebnisse hinsichtlich einer ergonomischen Verhaltensmodifikation erreicht (Abb. 6). Während mit einer visuellen Rückmeldung die mittlere Dauer bis zu einer Korrektur einer unergonomischen Haltung ca. $4 \mathrm{~s}$ beträgt, wird die Korrektur einer unergonomischen Haltung bei der taktilen Rückmeldung bereits in weniger als $2 \mathrm{~s}$ vom Mitarbeitenden eingeleitet.

Der Grund liegt darin, dass im taktilen Fall keinerlei Umkodierungsaufwand entsteht und die über die taktile Modalität übermittelte Ortsinformation exakt mit der räumlichen Information der durchzuführenden Tätigkeit übereinstimmt. Wickens (1984) oder Bubb (2015, S. 276) sprechen in diesem Fall von sogenannten kontaktanalogen Displays. Sie sind dadurch gekennzeichnet, dass sie die Informationsdarstellung kompatibel zur geforderten Handlung darstellen und Information genauso repräsentiert ist, dass keinerlei Interferenz auftritt und das Initiieren einer Handlung keinerlei Umkodierungsaufwand benötigt.

In der auditiven Bedingung sind zumindest die Kodierungen zwischen Körperhaltung und Rückmeldesystem voneinander getrennt. Das Rückmeldesystem nutzt die auditive Modalität und ist zeitlich kodiert, das Ansteuern des 


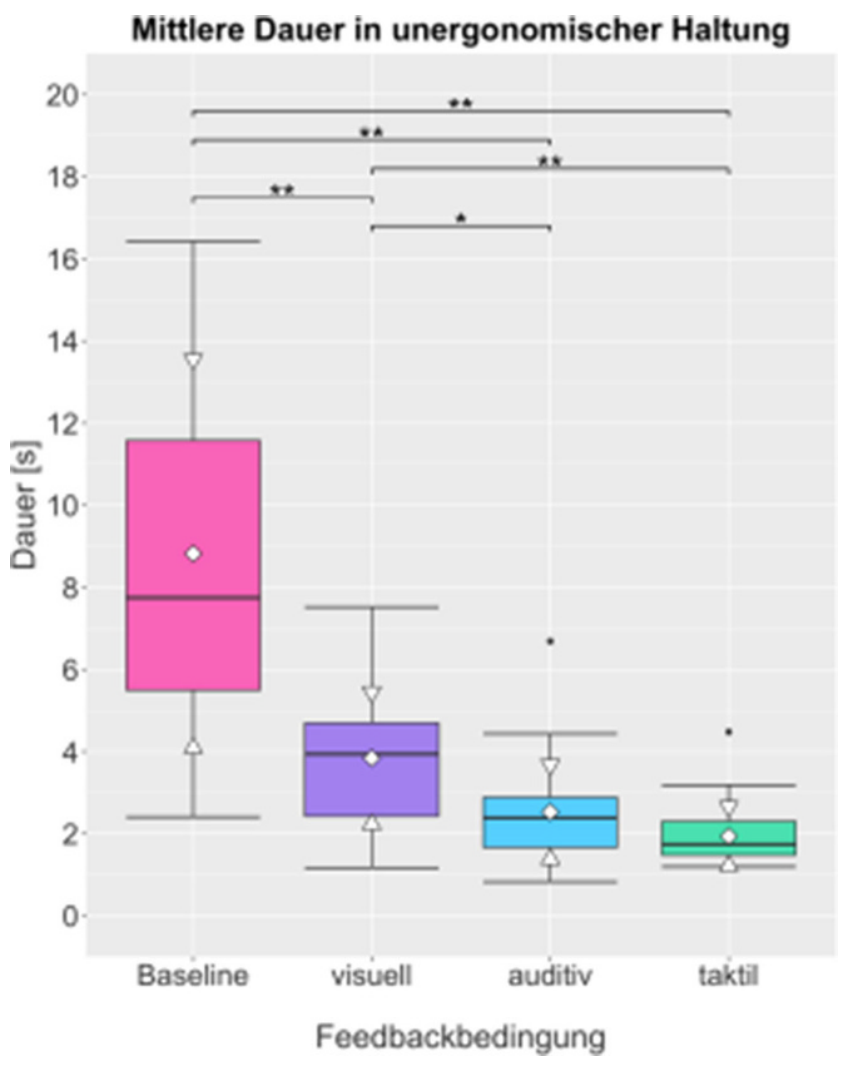

Abb. 6 Mittlere Dauer in nicht-ergonomischer Haltung abhängig von der Modalität der Rückmeldung. (Aus Burkhardt 2019, S. 181)

Fig. 6 Average duration in a non-ergonomic posture depending on the modality of the feedback. (From Burkhardt 2019, S. 181)

Muskel-Skelett Systems erfordert die taktile Modalität und räumliche Kodierung. Demzufolge liegt hier auf der Ebene der Kodierung keine Interferenzerscheinung vor. Der kognitive Aufwand besteht in diesem Fall darin, die auditive Information über Abruf der Gedächtnisinhalte inhaltlich zu interpretieren und das entsprechende muskuläre System anzusprechen.

In der visuellen Bedingung herrscht Interferenz vor, denn die über die Datenbrille übermittelte räumliche Information (über visuelle Modalität) muss auf die räumliche Information (über taktile Modalität) projiziert werden. Die Interferenz und der Umkodierungsaufwand resultieren hier aus dem Aufwand, die visuell räumliche Kodierung über die Gedächtnisinhalte auf die taktil räumliche Kodierung zu projizieren.

\section{Diskussion}

Das Konzept der Kompatibilität beschreibt wesentliche Eigenschaften des Menschen, wie er Information bei der Aufnahme strukturiert, nämlich abhängig von den Sinnesmodalitäten sowie einer zeitlichen und räumlichen Ordnung.
Der Zusammenhang von Kompatibilität mit der Ressourcentheorie und physiologischen Sachverhalten macht deutlich, dass diese Eigenschaften wesentliche Strukturierungshilfen sowie nicht veränderbare Eigenschaften der menschlichen Informationsverarbeitung sind. Diese Eigenschaften müssen von jedem technischen System, mit dem Menschen interagieren folglich berücksichtigt werden, um eine gute ergonomische Gestaltung zu erreichen. Kompatibilität ist damit ein wesentliches Element eines menschenorientierten Gestaltungsansatzes.

Auf Basis des Zusammenhangs der Ressourcentheorie mit dem Konzept der Kompatibilität kann festgehalten werden, dass Kompatibilität auch eine wesentliche Anforderung zur Reduktion kognitiver Belastung in Arbeitssystemen darstellt. Durch Kompatibilität wird der Umkodierungsaufwand reduziert und Interferenzen in kognitiven Tätigkeiten vermieden. Damit hat Kompatibilität auch für die derzeit viel diskutierte Bedeutung der psychischen Belastung eine besondere Wichtigkeit hinsichtlich der Auslegung von (digitalen) Systemen oder Arbeitsprozessen. Man kann sicher auch die Hypothese aufstellen, dass ein Großteil der psychischen Leistung durch die hohe Anzahl an inkompatiblen Gegebenheiten im Arbeitsprozess zustande kommt.

Das Konzept der Kompatibilität stammt aus einer Forschungstradition nach dem Zweiten Weltkrieg. Auf Basis der in diesem Paper dargestellten Zusammenhänge und insbesondere der Tatsache, dass Kompatibilität eng mit der Physiologie der Informationsverarbeitung zusammenhängt, muss abschließend herausgestellt werden, dass die Bedeutung der Kompatibilität für die Arbeitsgestaltung zeitlos ist (zumindest solange sich die Architektur unseres Gehirns nicht wesentlich verändert). Deshalb sind die Anforderungen der Kompatibilität für aktuelle Fragestellungen der Mensch-Roboter-Kollaboration (MRK), des Einsatzes von Head-Mounted-Displays (HMD) in der Montage oder Fertigung oder für die Gestaltung von Nutzerkonzept beim autonomen Fahren höchst aktuell. Um Information möglichst fehlerfrei und aufwandsarm erfassen zu können, ist es auch in solchen modernen Arbeitsgestaltungen erforderlich, die Grundprinzipien der Kompatibilität einzuhalten. Die gezeigten Beispiele demonstrieren deutlich, dass Kompatibilitätsprobleme auch in modernsten Arbeitssystemen von Bedeutung sind und eine gute Gestaltung zur Minimierung des Umkodierungsaufwandes die belastungsoptimalen Lösungen ergibt.

Funding Open Access funding enabled and organized by Projekt DEAL.

Open Access Dieser Artikel wird unter der Creative Commons Namensnennung 4.0 International Lizenz veröffentlicht, welche die Nutzung, Vervielfältigung, Bearbeitung, Verbreitung und Wiedergabe in jeglichem Medium und Format erlaubt, sofern Sie den/die ursprünglichen Autor(en) und die Quelle ordnungsgemäß nennen, einen Link 
zur Creative Commons Lizenz beifügen und angeben, ob Änderungen vorgenommen wurden.

Die in diesem Artikel enthaltenen Bilder und sonstiges Drittmaterial unterliegen ebenfalls der genannten Creative Commons Lizenz, sofern sich aus der Abbildungslegende nichts anderes ergibt. Sofern das betreffende Material nicht unter der genannten Creative Commons Lizenz steht und die betreffende Handlung nicht nach gesetzlichen Vorschriften erlaubt ist, ist für die oben aufgeführten Weiterverwendungen des Materials die Einwilligung des jeweiligen Rechteinhabers einzuholen.

Weitere Details zur Lizenz entnehmen Sie bitte der Lizenzinformation auf http://creativecommons.org/licenses/by/4.0/deed.de.

\section{Literatur}

Airsafe (2002) Fatal events since 1970 for British midland. Airsafe.com, LLC. http://airsafe.com/events/airlines/midland.htm. Zugegriffen: 1. Sept. 2021

Arenius M, Klippert J, Ott A, Sträter O (2013) Ceyeberman: Ein Messsystem zur Ermittlung der Arbeitsbelastung [Ceyberman: A System for Measuring Physical and Cognitive Workload]. Eisenbahningenieur 64(7):36-39

Bubb H (1993) Systemergonomie. In: Schmidtke H (Hrsg) Ergonomie. Hanser, München

Bubb H (2015) Systemergonomie des Fahrzeugs. In: Bubb H, Bengler K, Grünen RE, Vollrath M (Hrsg) Automobilergonomie. Springer, Heidelberg, S 259-344

Bubb H, Sträter O (2006) Grundlagen der Gestaltung des Mensch-Maschine-Systems. In: Zimolong B, Konrad U (Hrsg) Ingenieurspsychologie. Enzyklopädie der Psychologie. Hogrefe, Göttingen

Burghardt T (2019) Ergonomieassistenz - Entwicklung und Evaluierung von Assistenzkonzepten in der visuellen, auditiven und taktilen Modalität zur Verbesserung ergonomischen Verhaltens. Universität Kassel, Kassel. https://kobra.uni-kassel.de/handle/ 123456789/11270. Zugegriffen: 31. Dez. 2021

Burghardt T, Sträter O (2017) PREFLOW-Preventive Workflows: Intelligentes Assistenzsystem zur präventiven Steuerung von Arbeitsprozessen. Schlussbericht. Universitäts- und Technische Informationsbibliothek (TIBKAT), Hannover

Gentner D, Stevens A (1983) Mental models. Erlbaum, Hillsdale

Günebak S, Sträter O, Allgaier S (2010) Systemgestaltung durch Kombination mentaler Belastungsanalyse und Blickbewegungsaufzeichnung. GfA Frühjahrskongress 2010, Neue Arbeits- und Lebenswelten gestalten, Darmstadt, 24.-26. März 2010

Klippert J, Ott A, Jennerich M (2011) Effizienz und Reliabilität eines Systems zur computerunterstützten Haltungsanalyse. In: GfA (Hrsg) Mensch, Technik, Organisation - Vernetzung im Produk- tentstehungs- und -herstellungsprozess. GfA-Press, Dortmund, S $615-618$

Low I (2003) Assessment of the impact on mental workload from advanced air traffic management systems: a diagnostic tool. IEA 2003, Seoul/

Metternich J, Sträter O (2020) (Hrsg.). Digitale Assistenz für die Produktion. Ein Leitfaden für die Bedarfsermittlung, Gestaltung und Einführung. VDMA, Frankfurt

Sheridan TB (2002) Human and automation: System design and research issues. John Wiley \& Sons, New York. ISBN 0-47123428-1.

Spanner B (1993) Einfluß der Kompatibilität von Stellteilen auf die menschliche Zuverlässigkeit. VDI Reihe Biotechnik. Nr. 89. VDI, Düsseldorf

Strasser H (1993) Ergonomie - Arbeitsplatz. Kap. 2.4.5. Kompatibilität. In: Hettinger TH, Wobbe G (Hrsg) Kompendium der Arbeitswissenschaft. Kiel-Verlag, Ludwigshafen, S 228-242

Strasser H (2008) Kompatibilität, Leitlinie für ergonomische Arbeitsgestaltung und präventiven Arbeitsschutz. In: Produkt- und Produktions-Ergonomie - Aufgabe für Entwickler und Planer Proceedings des 54. Kongresses der Gesellschaft für Arbeitswissenschaft. GfA-Press, Dortmund, S 17-28

Strasser H (2021) Compatibility as guiding principle for ergonomics work design and preventive occupational health and safety. Z Arb Wiss. https://doi.org/10.1007/s41449-021-00243-0

Sträter O (2005) Cognition and safety-an integrated approach to systems design and performance assessment. Ashgate, Aldershot

Sträter O (2021) Entgrenzte Arbeitsgestaltung - Entgrenzen wir uns selbst? In: Hoppe A (Hrsg) Entgrenzte Welten. Herbstfachtagung Arbeit und Technik der kooperativen Forschungsstelle Technikstress. TU Cottbus. Cottbus

Sträter O, Bengler K (2019) Positionspapier Digitalisierung der Arbeitswelt. Z Arb Wiss 73:252-260

Sträter O, Bubb H (2003) Design of systems in settings with remote access to cognitive performance. In: Hollnagel E, Suparamaniam N (Hrsg) Handbook of cognitive task design. Lawrence Erlbaum, Hillsdale, S 333

Sträter O, Schmidt S, Stache S, Saki S, Wakula J, Bruder R, Ditchen D, Glitsch U (2018) Abschlussbericht Forschungsvorhaben „U-Linien-Montage-systeme“ U-Linien-Montagesysteme - Instrumente zur Gefährdungsbeurteilung und arbeitswissenschaftliche Gestaltungsempfehlungen zur Prävention. BGHM, Düsseldorf

Wickens CD (1984) Engineering psychology and human performance. C. E. Merrill Publishing Comp.any, A Bell \& Howell Company, Columbus

Wikipedia (Hrsg) (2021) Kompatibilität (Technik) - Wikipedia. https:// de.wikipedia.org/wiki/Kompatibilit\%C3\%A4t_(Technik). Zugegriffen: 1. Sept. 2021 